<smiles>[C+]1C=[C-]C=C1</smiles>

\title{
Comunicación asertiva entre padres y adolescentes
}

Assertive communication between parents and teens

Comunicação assertiva entre pais e adolescentes

Leidy Guevara

Universitaria Iberoamericana, Bogotá (Colombia) https://orcid.org/0000-0003-2705-6357

Nidia Pinzón

Universitaria Iberoamericana, Bogotá (Colombia) https://orcid.org/0000-0001-5064-0709

Marlem Osorio

Universitaria Iberoamericana, Bogotá (Colombia) https://orcid.org/0000-0002-1660-0372

D0I: https://doi.org/10.35622/j.rep.2021.04.003

Recibido 30/03/2021/ Aceptado 16/11/2021

RESUMEN. En las familias, la comunicación es un aspecto muy importante y fundamentale en el desarrollo de los jóvenes, de allí parte la confianza entre padres e hijos, desde esta perspectiva la interacción hace parte de dicha formación, es aquí donde se puede perder el respeto mutuo y los valores que deben enraizar las buenas relaciones. Surge la necesidad de comprender las experiencias de comunicación asertiva entre padres y adolescentes de 12 a 16 años en la localidad de Ciudad Bolívar Bogotá. Se utiliza una metodología cualitativa con técnica de análisis fenomenológico. La población está conformada por 200 estudiantes en etapa de la adolescencia entre los grados 7 a 11. Se tomó una muestra de 10 estudiantes, cinco de sexo masculino y cinco de sexo femenino. Con el microanálisis y codificación abierta se identifican 27 códigos relevantes para la investigación, agrupadas en seis familias de códigos para ser analizados: construcción de la personalidad, fortalecimiento de los vínculos afectivos, malas conductas, habilidades comunicativas y técnicas para gestionar conflictos. Se concluye que la relación entre padres y adolescentes debe ser enfatizada en aquellos valores y fortalecimiento de los lazos familiares, como también el asertividad que conlleva a las buenas prácticas conductuales de los adolescentes.

PALABRAS CLAVE: Comunicación asertiva, adolescentes, familia, padres.

ABSTRACT. In families, communication is an essential and fundamental aspect in the development of young people, hence the trust between parents and children; from this perspective, interaction is part of said training; this is where mutual respect can be lost and the values that good relationships should root. Therefore, the need arises to understand the assertive communication experiences between parents and adolescents from 12 to 16 years old in Ciudad Bolívar Bogotá. Therefore, a qualitative methodology is used with a phenomenological analysis technique. The population is made up of 200 students in the adolescence stage between grades 7 to 11 . A sample of 10 students were taken, five male and five female. With microanalysis and open coding, 27 relevant codes for research are identified, grouped into six families of principles to be 
analyzed: construction of personality, strengthening of affective bonds, destructive behaviors, communication skills, and techniques to manage conflicts. It is concluded that the relationship between parents and adolescents should be emphasized in those values and strengthening of family ties and the assertiveness that leads to good behavioral practices of adolescents.

KEYWORDS: Assertive communication, adolescents, family, parents.

RESUMO. Nas famílias, a comunicação é um aspecto muito importante e fundamental no desenvolvimento dos jovens, daí a confiança entre pais e filhos, nesta perspectiva a interação faz parte dessa formação, é aqui que se pode perder o respeito mútuo e os valores que bons relacionamentos devem enraizar. Surge a necessidade de compreender as experiências de comunicação assertiva entre pais e adolescentes de 12 a 16 anos na cidade de Ciudad Bolívar Bogotá. É utilizada uma metodologia qualitativa com uma técnica de análise fenomenológica. A população é composta por 200 alunos na fase da adolescência, entre $7^{\mathrm{a}}$ e $11^{\mathrm{a}}$ séries. Foi coletada uma amostra de 10 alunos, cinco do sexo masculino e cinco do feminino. Com microanálise e codificação aberta, são identificados 27 códigos relevantes para a pesquisa, agrupados em seis famílias de códigos a serem analisados: construção da personalidade, fortalecimento de vínculos afetivos, comportamentos inadequados, habilidades de comunicação e técnicas de gestão de conflitos. Conclui-se que a relação entre pais e adolescentes deve ser enfatizada naqueles valores e no fortalecimento dos laços familiares, bem como na assertividade que leva às boas práticas comportamentais dos adolescentes.

PALABRAS CLAVE: Comunicação assertiva, adolescentes, família, pais.

\section{INTRODUCCIÓN}

La comunicación es una de las formas más comunes que se encuentran en el desarrollo de los seres humanos porque a partir de ella se expresa y se dan a conocer ideas que se forman dentro de la sociedad. Desafortunadamente esta comunicación ha escaseado en algunos de los hogares, lo que evidencia ciertos tipos de conflictos tanto personales como individuales. Situación que influye en el estado emocional y vivencial de los adolescentes, razón por la que se buscó identificar aquellas experiencias de comunicación asertiva entre padres y adolescentes, incidiendo en las relaciones que se llevan a cabo dentro del contexto.

La adolescencia es la etapa en la que los jóvenes tratan de individualizarse y profundizar en lo que les entretiene e interesa dejando de lado el espacio familiar. Esta actitud suele venir precedida por la falta de comunicación dentro del entorno que los rodea o por el mal uso de la misma. Es por ello, que es indispensable fomentar la comunicación, para el buen desarrollo personal y social del adolescente.

Es importante mencionar que el tema de investigación ayuda al desarrollo de los individuos y sus comportamientos comunicacionales dentro y fuera del núcleo familiar, por tanto, se ve un aporte a la sociología y a la propia comunicación y sus ramas de estudio. La comunicación asertiva es una estrategia que se ubica en el medio de dos conductas que resultan opuestas: la pasividad y la agresividad. 
Según Caridad et al., (2017) expresan que: "la comunicación asertiva es aquella en la que está presente un adecuado equilibrio entre la agresividad en un extremo y la no afirmativita en el otro, donde se integra el respeto por sí mismo y por los demás" (p.120).

Por otro lado, ayuda a determinar cómo funciona la percepción y cómo interactúan los individuos con los mensajes; así como también entender las conductas de los adolescentes y los padres. Esta investigación estimula a que cada miembro de la familia aprenda a dar un aporte positivo y de superación a cada uno de los conflictos que se pueden presentar en el hogar, solucionándolo de manera asertiva y rápida. Cáceres (1991) expresa "cuando la familia utiliza medios de comunicación funcional se logra: la responsabilidad de la sociabilización de los niños, se satisfacen las necesidades emocionales de sus miembros, se mantiene la relación conyugal y se participa activamente en la sociedad" (s.p). Se entiende entonces, que la comunicación funcional actúa de base para el buen relacionamiento del individuo dentro del núcleo familiar, garantizando que este vínculo entre individuos también sea óptimo en el ámbito social.

Por lo que se puede decir que, con esta investigación, se está aportando a la disminución de ciertas conductas y situaciones conflictivas, por medio de la comunicación asertiva, clara, respetuosa y honesta entre padres e hijos. Se evita el aumento de conflictos dentro del hogar, creando lazos de confianza y fortaleciendo la relación familiar lo cual les permitirá emitir conductas positivas sobre el sistema social y familiar. La comunicación es un proceso innato en el ser humano, es una necesidad básica que se determina biológicamente lo cual hace que las personas sean seres sociales. Desde que se nace el proceso de interacción es la comunicación y este se desarrolla a través de diferentes códigos, hace parte de las relaciones humanas en el que dos o más participantes intercambian un mensaje mediante la expresión o el lenguaje, lo que se percibe como un proceso interactivo y social.

El psicoterapeuta Watzlawick (1921) indica que "la comunicación ejerce un papel fundamental en nuestras vidas y en el orden social, aunque apenas seamos consciente de ello" (p.412). Es por ello que la comunicación es el intercambio de información que se origina en una relación, lo importante no es tanto el modo de comunicarnos o si este es consecuente o no, sino el cómo se transmite la información y de qué manera esta influye unos a otros. 
A partir de esto, se puede incluir la Teoría de la semiótica que tiene como objeto de interés el estudio de los signos de la vida social. Se encarga de analizar la presencia de estos signos dentro de la sociedad, es un concepto muy parecido al de semiología, en pocas palabras, se define como la ciencia que estudia las propiedades generales de los sistemas, elementos esenciales en la interacción y supervivencia de todo ser humano. Para Eco (2000), expresa que la semiótica tiene un solo deber, definir al sujeto de la semiosis mediante categorías exclusivamente semióticas, porque es a partir de ella que "los individuos empíricos comunican y los sistemas de significación hacen posibles los procesos de comunicación" (p. 424).

La semiótica estudia todos los procesos culturales como procesos de comunicación y tienden a demostrar que bajo los procesos culturales existen sistemas. Eco (2000) concluye que, "por un lado, se ha tendido a la descripción de cada sistema semiótico como si fuera un sistema cerrado, rigurosamente estructurado y visto en un corte sincrónico" (p. 374). También menciona que: "el modelo comunicativo de un proceso abierto, en el que el mensaje varía según los códigos, actúa según las ideologías y circunstancias. El sistema de signos se reestructura sobre la base de la experiencia de descodificación (semiosis in progress)" (p.374).

Por tanto, se puede decir que los signos, sirven para otras cosas que solo para adquirir conocimiento, y la semiótica es más amplia que el estudio del lenguaje de la ciencia. La semiótica proporciona una base para la comprensión de las principales formas de comunicación de la actividad humana y para su interrelación, puesto que todas estas actividades y relaciones se reflejan en los signos que median esas actividades.

Morris (1938), expone que, "la semiótica es una ciencia que estudia cosas o sus propiedades, su función es servir como signos, también es el instrumento de la totalidad de las ciencias, cada ciencia utiliza y expresa sus resultados por medio de signos" (p. 25). Se entiende que la civilización humana depende de los signos y los sistemas de signos ve a la semiótica como la ciencia que se encarga de su estudio. La semiótica funciona como un instrumento unificador pues aporta fundamentos especiales en los signos. Un signo, por tanto, es una cosa que está en lugar de otra, para alguien bajo ciertos aspectos o 
circunstancias, además crea en la mente de esa persona un signo equivalente.

Para Peirce (1945) todo lo que existe es signo, "tiene capacidad de ser representado, mediar y llevar ante la mente una idea. En ese sentido, es el estudio del más universal de los fenómenos y no se limita a un simple estudio y clasificación de los signos". (p. 98). A partir de lo anterior se puede deducir que se utiliza la categoría de interpretante para nombrar ese proceso, por el cual se elige un signo equivalente en la mente. El signo se presenta como el resultado de la unión entre tres elementos: el objeto que lo determina, el signo material que lo expresa y el signo que lo interpreta y es a partir de dichos elementos que se le denomina semiosis.

Son muchos los autores que han aportado al conocimiento de la semiótica, sin embargo, Sanders (1945) ha sido considerado como "padre" de la semiótica. Proporciona una teoría general completa del significado y la representación de signos, por lo que la semiótica ha tenido como fin explicar la significación a través del estudio de los sistemas de signos. Al interior de la disciplina, el signo permite entender la forma en que los individuos dan significado al mundo, y en todos los casos se trata de una representación que da a entender otra cosa. Sin duda alguna la semiótica y la comunicación es uno de los pilares básicos de nuestra sociedad y el lenguaje es el medio de expresión que utilizamos en todo acto comunicativo.

Son muchos los lingüistas y filósofos que coinciden al identificar pensamiento y lenguaje, pues consideran que el pensamiento está verbalizado. Es evidente que el ser humano necesita un instrumento que organice y estructure su propio pensamiento para alcanzar un desarrollo global, es decir, a nivel comunicativo-lingüístico, cognitivo y psicosocial; de lo contrario, el pensamiento sin dicho instrumento, sin lenguaje, es algo sin forma, sin sentido, lo cual suele derivar al retraso mental o a la imposibilidad de un desarrollo cognitivo normal.

De acuerdo con Watzlawick (1985) la comunicación humana está compuesta por tres áreas, la sintáctica, la semántica y la pragmática, la primera hace referencia a la transferencia del mensaje, la segunda se interesa en el significado o esencia del mensaje que se transfiere y la pragmática se centra en la afectación que la comunicación tiene sobre la conducta 
humana. El autor introduce los conceptos de: conciencia o inconsciencia de los actos, presente versus pasado, efectos versus causas, la circularidad de las pautas de la comunicación, la relatividad de lo "normal" y lo "anormal" los cuales sirven de antecesores de los axiomas.

Se puede decir entonces, que la comunicación es indispensable en la vida humana y que diversos enfoques la conciben como un ritual que los seres humanos llevan a cabo desde que nacen y ello se determina a través de cinco axiomas de la comunicación expuestos en la Teoría de la Comunicación Humana de Watzlawick (1985) que son reseñados a continuación:

La imposibilidad de no comunicar: Toda interacción con el otro trae consigo un mensaje que movilizará la conducta de los participantes, comunicarse no es sólo hablar o no hablar. Todo acto que hacemos, o incluso los que no hacemos, tiene un significado que puede ser percibido o interpretado y alterar el comportamiento de los receptores. Watzlawick (1985) indica que,

Lo que significa que la comunicación no es solo verbal sino también gestual - no verbal. Este axioma interpreta todo comportamiento o conducta como mensaje comunicacional que puede ser interpretado y dotado de significado por alguien que se encuentre en el sistema (p.51).

Niveles de contenido y relaciones de la comunicación: El mensaje transmitido va a ser interpretado por el oyente o el receptor en función a la relación que mantenga con el emisor. Watzlawick (1985) expresa que "toda comunicación tiene un aspecto de contenido o semántico y un aspecto relacional, tales que el segundo clasifica al primero y es, por ende, una metacomunicación" (p.52).

La puntuación de la secuencia de hechos: La comunicación se da de manera bidireccional, donde se interactúa en una secuencia definida por el emisor y el receptor. En ocasiones dicha estructura se ve mediada por la respuesta al comportamiento del otro, ampliando o modificando el ciclo comunicacional, Según Watzlawick (1985) "no se reduce explícitamente a una causa-efecto. Puesto que, la naturaleza de la relación depende de la puntuación (organización) de las secuencias de comunicación entre los comunicantes" (p.56). 
Comunicación digital y analógica: La comunicación digital y analógica se complementan, ya que el aspecto digital del mensaje se codifica en función de lo analógico, lo digital hace referencia a la comunicación verbal, medible, y lo analógico a todo lo que incluye la comunicación no verbal, es decir hay que valorar tanto las palabras como otros aspectos tales como los gestos, el tono, la distancia y la posición: Watzlawick (1985) refiere este axioma como:

"El lenguaje digital cuenta con una sintaxis lógica sumamente compleja y poderosa, pero carece de una semántica adecuada en el campo de la relación mientras que el analógico posee la semántica, pero no una sintaxis adecuada para la definición inequívoca de la naturaleza de las relaciones" (p.61)

Interacción simétrica y complementaria: El último de los axiomas propuestos es especialmente relevante en el ámbito organizacional, y establece que hay que tener en cuenta que puede haber relaciones de simetría o complementariedad en las relaciones comunicativas, según si todos tienen el mismo rol o posición de poder o bien dicha relación es desigual. Watzlawick (1985) expresa que "la interacción simétrica se caracteriza por la igualdad y por la diferencia mínima, mientras que la interacción complementaria está basada en un máximo de diferencia" (p.69).

Por tal razón desde una perspectiva sistémica se plantea que toda situación interpersonal es comunicación, puesto que la comunicación es el vehículo de las manifestaciones observables, de la relación que existe entre los individuos, sea esa relación de amor, odio, rabia o respeto. Es por ello que, el principio de circularidad complementa el proceso secuencial de acción y reacción de las conductas comunicacionales que tienen lugar en un periodo de tiempo y en un contexto espacial, siguen un patrón de circularidad, de modo que la conducta comunicacional de cada persona afecta a la de los otros y es, a su vez, influida por la de los demás.

Por otra parte, se asume desde la teoría de sistemas que la repetición de secuencias comunicacionales es de gran importancia de las relaciones que aparecen en sistemas duraderos como la pareja, los amigos o la familia. Los participantes intentan definir con sus patrones de comunicación la naturaleza de su relación con el otro. De hecho, adaptan su respuesta en función de su propia definición de la relación confirmando, rechazando o 
modificando la definición del otro. Así, se establecen unas reglas de relación que regulan la conducta de los participantes.

Bertalanffy (1987) definió a un sistema como una serie de elementos interrelacionados con un objetivo común, que se afectan unos a otros, y la característica que los une es la composición que tienen. Es decir, la totalidad, la cual no es sólo la suma de las partes, sino también la relación entre ellas. Además, cada elemento tiene una función e interactúan entre ellos.

Según Oslon (1991) "la comunicación es la dimensión facilitadora y crítica para que haya cohesión y adaptabilidad en la familia". De aquí que las habilidades de comunicación positiva, tales como empatía, escucha reflexiva y apoyo, faciliten a las parejas y familias compartir entre sí sus necesidades y preferencias cambiantes con respecto a la cohesión y a la adaptabilidad. Feixas et al., (2012) aluden el enfoque sistémico como:

El sistema familiar, como un todo, como un organismo estructurado e interdependiente que se comunica con unas pautas de interacción, y en las que el individuo sólo es uno de sus componentes, su valor tiene que ver con la función y posición en el sistema (p.3).

La posición de la teoría de los sistemas y según el enfoque general que se adopte se pueden encontrar sistemas dentro de sistemas más amplios o generales, los cuales influencian a los que los forman. Para Bronfenbrenner (1979) plantea la teoría ecológica de sistemas, en la que afirma la existencia de múltiples sistemas que afectan directamente el desarrollo de la persona durante la vida.

A partir de lo anterior se puede decir que la comunicación es indispensable en la vida humana y que diversos enfoques la conciben como un ritual que los seres humanos llevan a cabo desde que nacen y ello se determina a través de cinco axiomas de la comunicación expuestos en los sistemas que envuelven al individuo son entendidos en el entorno como un conjunto de sistemas correlacionados, así se identifican cinco diferentes y que son expuestos en el libro La Ecología del desarrollo humano de Bronfenbrenner (1979), que son descritos a continuación: 
El Microsistema (entorno inmediato como la familia y el hogar), es el nivel más cercano y estaría constituido por los padres, la familia y la escuela. La relación entre este y el desarrollo es más que evidente. Por ejemplo, las creencias de los padres van a afectar de manera directa a cómo acabará siendo éste. La familia tiene un papel central en el desarrollo emocional y la escuela en la socialización. Esta relación es bidireccional, es decir, la forma en la que se comporten influirá en el entorno.

El Mesosistema (relaciones entre microsistemas, como los padres y los profesores), cómo se relacione la familia con los profesores o los padres con los amigos del adolescente. De ahí la importancia de los vínculos que establezcan los padres con el resto de los entornos.

El Exosistema (más extenso, como los recursos del barrio, la localidad o la ciudad), estaría compuesto por los elementos que afectan a la vida del adolescente pero que no tienen una relación directa con él, sino que esta se produce de manera indirecta a través de los miembros que forman el microsistema.

El Macrosistema (los valores culturales y políticos de la sociedad). Además, la familia puede ser descompuesta en distintos subsistemas como son el parental (padre-hijo), el conyugal (padres) y el fraternal (hermanos). Este nivel estaría definido por elementos de la cultura, las tradiciones, el sistema de valores de su sociedad o la religión.

Desde el punto de vista sistémico es muy importante trabajar atendiendo, en la medida de lo posible, a todos los sistemas involucrados en la vida de la persona. La prioridad está en la intervención en el sistema más cercano y estable en la vida del individuo y este acostumbra a ser el familiar. La teoría de la comunicación ha aportado a la configuración de la terapia familiar sistémica desde el análisis de interacción. García (2011), expresa que:

Las reglas que guían las diferentes formas de interacción social, las aportaciones de Palo Alto expuestas, sobre todo, en teoría de la comunicación humana, se han aplicado de forma práctica al campo de las terapias sistémicas, donde cada interacción debe ser tratada como promulgaba como un sistema abierto en intercambio de informaciones con su entorno. Por lo anterior, puede decirse que la teoría de la comunicación humana es hoy un clásico dentro del campo de la 
terapia sistémica, pues permitió el abandono del clásico análisis de los procesos psíquicos intraindividuales sustituyéndolo o complementándolo con el análisis de los procesos de interacción-relación comunicacional. En otros términos, esta obra propició el tránsito del foco centrado en la psique individual al foco centrado en el sistema interaccional (p. 6-7).

Es entonces, que la Teoría de la Comunicación y la Teoría de los Sistemas tienen puntos de encuentro y en este caso marca un aspecto importante ya que conceptualmente la Terapia Familiar se nutre de la teoría de la comunicación, especialmente con los axiomas propuestos por Watzlawick (1967) como herramienta para el trabajo terapéutico. En pocas palabras, se define como la ciencia que estudia las propiedades generales de los sistemas, elementos esenciales en la interacción y supervivencia de todo ser humano.

Por tal motivo la Terapia Familiar Sistémica y los Axiomas de la Comunicación Humana tienen en común un enfoque sistémico, en los cuales se tiene en cuenta el principio de totalidad y de causalidad circular, la interrelación e interdependencia; también comporten de base el estudio de las interacciones humanas, la interrelación y la conexión, no se puede hacer Terapia Sistémica sin hacer uso de los Axiomas de la Comunicación, por todo lo anterior es importante poder Comprender las experiencias de comunicación asertiva entre padres y adolescentes de 12 a 16 años en la localidad de Ciudad Bolívar Bogotá.

\section{MATERIAL Y MÉTODOS}

\section{Tipo de estudio y Técnica de Análisis}

Se optó por una metodología de carácter cualitativo, pues a partir de ella se puede acceder a la población llegando a una descripción completa y detallada que proporcione una investigación más clara. Por medio del enfoque metodológico cualitativo se puede llegar a comprender cada una de las necesidades y peculiaridades que hacen que los escenarios sean observados a partir de lo que dicen, hacen y sienten. Dentro de la investigación cualitativa existen ciertas características que sintetizan los criterios definitorios de la misma, los cuales conllevan a que ésta sea inductiva, holística, sensible y comprensiva que busca identificar y experimentar la realidad y cuáles son los métodos humanistas. Este método se adapta y facilita la recogida de datos empíricos que ofrecen descripciones complejas de acontecimientos, interacciones, comportamientos, pensamientos, conduciendo al 
desarrollo o aplicaciones de categorías y relaciones que permiten la interpretación de los datos. En este sentido, se hace necesario que los datos sean integrados e interpretados de forma narrativa y que sea el observador quien llegue a sus propias conjeturas.

Frente a los métodos de investigación y análisis de datos se conlleva a la perspectiva de la técnica de análisis fenomenológica la cual busca comprender los fenómenos sociales desde la propia perspectiva del actor, comprendiendo así el contexto personal los motivos y creencias que están detrás de las acciones, es así como la técnica de análisis fenomenológico no tiene límites, por lo que puede estudiarse todo tipo de emociones, experiencias, razonamientos o percepciones.

\section{Población}

La población objeto de estudio, se encuentra en la localidad de Ciudad Bolívar en la ciudad de Bogotá de un establecimiento educativo público, con estudiantes en etapa de la adolescencia de 12 a 16 años entre los grados 7 a 11, con un total de 200 alumnos. La muestra entonces está conformada por el $10 \%$ que equivaldrían a 10 estudiantes, 5 de sexo masculino y 5 de sexo femenino, lo que permitirá recoger y representar una población para el adecuado análisis y estudio dentro del enfoque cualitativo.

\section{Instrumento de recolección de datos}

El instrumento de recolección de información utilizado en esta investigación es la entrevista en profundidad la cual busca una comunicación interpersonal establecida entre el investigador y el sujeto de estudio con el propósito de obtener respuestas verbales a los interrogantes planteados sobre el problema propuesto.

\section{Procedimiento}

El proceso de recolección y análisis de datos inicia con la fase 1 que consiste en indagar sobre la comunicación entre padres y adolescentes. Para esto se debe hacer una búsqueda de referentes, hacer la construcción del Marco Referencial, utilizando una rejilla bibliográfica. Seguido a esto, se encuentra la fase 2 que consiste en analizar los métodos de comunicación entre padres y adolescentes de 12 a 16 años en la ciudad de Bogotá Colombia, para lo cual, se debe hacer la transcripción, la codificación, confeccionar el guion, 
tener un acercamiento con la población y, por último, hacer la recolección de la información. Para esto se utiliza un formato de guion, el consentimiento informado y tabla de codificación.

\section{Consideraciones Éticas}

El Acuerdo 11 de la Res. 8430 de 1993 del Ministerio de Salud, por la cual se establecen las normas científicas, técnicas y administrativas para la investigación en salud. Del título II de la investigación en seres humanos, el capítulo 1 de los aspectos éticos se tiene que el Artículo 5 indica que en toda investigación en la que el ser humano sea sujeto de estudio, deberá prevalecer el criterio del respeto a su dignidad y la protección de sus derechos y su bienestar. Por lo que en esta investigación se da cumplimiento y garantía de tal consideración.

También se considera pertinente dar cumplimiento al Artículo 8 en el que se expresa que en las investigaciones en seres humanos se protegerá la privacidad del individuo, sujeto de investigación, identificándolo solo cuando los resultados lo requieran y éste lo autorice. En el presente estudio se respetará el derecho a la privacidad y al anonimato si así lo desean los individuos objetos de estudio.

Igualmente se tiene que en concordancia con el Artículo 9, que considera como riesgo de la investigación la probabilidad de que el sujeto de investigación sufra algún daño como consecuencia inmediata o tardía del estudio. El Artículo 10 expresa que el grupo de investigadores o el investigador principal deberán identificar el tipo o tipos de riesgo a que estarán expuestos los sujetos de investigación. Se determina que en la presente investigación no se genera ningún riesgo de daño en la salud y seguridad en los individuos objetos de estudio, dado que no se realizan pruebas físicas.

Por lo tanto, esta investigación entra en la clasificación de la categoría del inciso a expuesta en el Artículo 11 que determina que es una investigación sin riesgos, ya que, las técnicas y métodos que se emplean en esta investigación como entrevistas y cuestionarios que no generan cambios, ni se hacen intervenciones o modificaciones en las características y conductas de los individuos objetos de estudio. Cabe resaltar que de igual forma se dará cumplimiento a los demás artículos del capítulo 1 de esta resolución. 


\section{RESULTADOS}

\section{Microanálisis y codificación abierta}

Dando desarrollo a la investigación y para dar respuesta a ¿Cuáles son las experiencias de comunicación asertiva entre padres y adolescentes de 12 a 16 años en la localidad de ciudad Bolívar Bogotá? Se han identificado 27 códigos que refieren a entrevistas relevantes para la investigación las cuales se han agrupado en 6 familias de códigos para ser analizados de la mejor manera, a partir de las trascripciones de las entrevistas a continuación se presenta un cuadro de la transcripción realizada:

Tabla 3. Distribución de códigos

\begin{tabular}{|c|c|c|}
\hline Códigos & Total códigos & Familia de códigos \\
\hline Constancia & 1 & \multirow{6}{*}{ 1. Construcción de la personalidad } \\
\hline Confianza & 20 & \\
\hline Respeto & 18 & \\
\hline Seguridad & 7 & \\
\hline Sinceridad & 8 & \\
\hline Comprensión & 5 & \\
\hline Afectividad & 4 & \multirow{4}{*}{$\begin{array}{l}\text { 2. Fortalecimiento de los vínculos } \\
\text { afectivos }\end{array}$} \\
\hline Tiempo & 5 & \\
\hline Acompañamiento & 1 & \\
\hline Dialogo & 15 & \\
\hline Manipulación & 1 & \multirow{5}{*}{ 3. Malas conductas } \\
\hline Mentiras & 2 & \\
\hline Conflictividad & 19 & \\
\hline Relaciones intermitentes & 4 & \\
\hline Recepción del mensaje & 7 & \\
\hline Transmisión del mensaje & 6 & \multirow{4}{*}{ 4. Habilidades comunicativas } \\
\hline Efectividad & 1 & \\
\hline Opinión & 2 & \\
\hline Lenguaje no verbal & 9 & \\
\hline Solución de conflictos & 14 & \multirow{5}{*}{$\begin{array}{l}\text { 5. Técnicas para gestionar } \\
\text { conflictos }\end{array}$} \\
\hline Buenas relaciones & 30 & \\
\hline Asertividad & 13 & \\
\hline Necesidades & 8 & \\
\hline Emociones & 16 & \\
\hline
\end{tabular}

Fuente: elaboración propia

Es así como, en el cuadro anterior se logra observar los códigos, el número de veces que se repite y la familia en la cual se agruparon, permitiendo captar las miradas de los entrevistados frente a la comunicación familiar como habitantes de la localidad de ciudad Bolívar. A partir de ello se da una descripción de cada familia de códigos. 
La familia de códigos "construcción personalidad" surge en la agrupación de aquellos códigos que permiten ver en los entrevistados los valores que los rodean en el entorno y la importancia que tienen en las relaciones sociales y familiares que surgen de la localidad de ciudad Bolívar es así como se evidencia que los entrevistados valoran la importancia de fortalecer los lazos familiares a través de las enseñanzas de los padres, como lo señalan los entrevistados cuando:

"Me parece que es necesaria la comunicación porque, pienso que facilita la relación entre mis padres y yo, pues la confianza es lo primero" (Entrevistado 1).

"Pienso que es importante la sinceridad, el respeto y el amor" (Entrevistado 5).

Además de la importancia de fortalecer los lazos familiares también se logra evidenciar que la familia es la que inculca dichos valores y ello se refleja en la interacción con el otro tal como se evidencia en el entrevistado cuando señala

"Tengo una actitud positiva y entablo una relación muy buena con cualquier persona diferente a mi edad, no soy esquiva, ni rechazo a nadie en el momento de dialogar o conversar con algunos de ellos" (Entrevistado 3).

A partir de ello también se visibiliza como el diálogo constante es importante dentro de las relaciones familiares y el mirar a los ojos cuando se habla es indispensable, al indicar que

"Este tipo de comunicación lo utilizamos constantemente, mis padres dicen que es importante hablar las cosas" (Entrevistado 3).

"Cuando nos miran a los ojos, sabemos que están prestando toda la atención necesaria, y podemos seguir el dialogo con más confianza" (Entrevistado 8).

Otro aspecto que se logra evidenciar en los relatos de los entrevistados es la diferencia que hay al relacionarse con amigos o padres, ello se refleja al preguntar ¿Cómo son las relaciones con sus padres y personas de su misma edad?

"Pues con mis padres es más seria y respetuosa que con mis amigos, con ellos recochamos más" (Entrevistado 6). 
"La relación con mis padres es buena y con mis amigos mucho mejor porque compartimos los mismos tipos de música y hablamos cosas de nuestra edad" (Entrevistado 2).

Para finalizar frente al código de construcción de personalidad se logra constatar como las familias son parte importante en el desarrollo de la personalidad del adolescente y como el compartir constantemente con ellos se refleja en la cotidianidad, tal y como los señalaron los siguientes entrevistados

"Son muy cálidas y se manejan siempre desde el respeto por la opinión del otro, mis padres son muy buenos oyentes y me gusta cuando estamos en la sala y compartimos una charla agradable de lo que les haya sucedido en el trabajo o en mis clases, otro sitio en el cual compartimos de una charla es el comedor y cuando vamos al parque o al centro comercial o la calle" (Entrevistado 3).

“......Por lo general siempre estoy con primos casi de mí misma edad y mis tíos son muy chéveres conmigo no tengo problemas por compartir con ellos" (Entrevistado 2)

..." Buena, nos reímos mucho y compartimos buenos momentos" (Entrevistado 6)

La familia de código "Fortalecimiento de los vínculos afectivos" hace referencia a los espacios y la disposición de los padres por interactuar con los entrevistados de la localidad de Ciudad Bolívar y como ello influye en fortalecimiento de la afectividad los cuales indican

"Cuando hablo con mis padres me siento seguro porque ellos me escuchan, me comprenden, me consuelan y me apoyan." (Entrevistado 9)

Otro factor que es visiblemente importante es el diálogo constante en los entrevistados que para varios de ellos es indispensable en los vínculos de padres e hijos pues el intercambiar opiniones y verbalizar sus problemas los hace sentir importantes, algunos expresaron que "Bueno pues el tipo de comunicación que utilizo es el dialogo constante con ellos, les tengo mucha confianza, en especial a mi mamá." (Entrevistado 1) 
"Son muy bonitas, tratamos de adelantar cosas de lo que sucede en la semana, y los espacios son el comedor o cuando salimos a departir en familia, un parque, centro comercial" (Entrevistado 2).

Con base en lo anterior se presume que los entrevistados constantemente necesitan ser escuchados y el hablar con los padres los satisface, como se evidencia en los relatos de acompañamiento, los cuales expresaron cosas como

"Heeeee, pues son tranquilas, me gusta aprender de las experiencias que han vivido mis padres, sé que ellos quieren lo mejor para mí y cumplo con mis deberes, así todo va bien. Hablamos siempre de que sea necesario en cualquier momento" (Entrevistado 9).

"Cuando los padres ven que un hijo está haciendo algo malo, o algo en lo que puede salir afectado y ellos deben intervenir, también se debe entablar cuando uno como hijo hace algo bueno y uno se siente orgulloso por algo, sentir el apoyo de los padres en esos momentos es importante" (Entrevistado 4).

Para ultimo frente al código de fortalecimiento en los vínculos afectivos se ve como los entrevistados hacen referencia al tiempo que se comparte en familia, siendo este un factor indispensable en el proceso de comunicación y que en ciertas ocasiones se queda corto como lo señalan cuando se les pregunta ¿Cómo son las conversaciones con sus padres y que espacios utilizan para ello? Los entrevistados responden

"Las conversaciones con mis padres son cortas, pues siempre tienen algo que hacer, solo cuando vamos de paseo podemos hablar más tiempo y nos sentimos súper bien" (Entrevistado 7).

"...Generalmente en la noche o los fines de semana, cuando hay más tiempo libre" ... (Entrevistado 8).

La familia de códigos "malas conductas" hace referencia a los comportamientos que pueden adquirir los entrevistados hacia los padres. En este código se evidencia la conflictividad que se puede dar por falta de comunicación de los padres hacia los hijos, los entrevistados 
señalan

"Un obstáculo puede ser la falta de confianza profe, si no hay ese tipo de confianza, no hay buena relación entre los hijos y los padres y por otra parte podrían ser los regaños, porque si los papas todo el tiempo regañan cada vez que cuantas algo, pues no volverían a hacerlo" (Entrevistado 1).

Otro elemento que se logra evidenciar en el código de familia de malas conductas es la manipulación, como lo indican uno de los entrevistados al decir que

"A veces en esta edad hablamos con mucha seguridad para lograr lo que queremos de nuestros padres. Jajajaja" (Entrevistado 8)

Otra situación que se agrupa en las malas conductas son las mentiras, que conllevan a conflictos, a los malos hábitos y que los entrevistados de la localidad de ciudad Bolívar infieren que no habría buenas relaciones

"Pienso que las barreras que pueden dañar una comunicación es la mentira hacia los padres, el mentir hace que se pierdan buenas relaciones" (Entrevistado 3)

Para concluir este grupo de familia, los entrevistados hacen referencia a situaciones de relaciones intermitentes, expresan que en ocasiones las relaciones se tornan pesadas, conllevando a que a veces están bien y otras no, ellos señalan

"Pues... mi mamá me dice que estoy llevando la contraria, quiero que me dejen hacer lo que yo quiera, y con mi hermano menor pues... a veces estamos bien cuando jugamos, pero cuando no quiero jugar peleamos" (Entrevistado 7)

"Pues, es buena cuando yo les digo que les voy a contar algo y quiero que me den su opinión, pero cuando mis padres se meten en mis cosas íntimas y tratan de imponer sus creencias ya se daña la comunicación" (Entrevistado 8).

La familia de códigos "Habilidades Comunicativas" hace referencia a los diferentes estilos de comunicación que manejan los entrevistados, y como a través de ellos las relaciones en 
familia son más eficaces y claras donde la recepción del mensaje se hace importante, señalando

“Ehhh.. por parte de mi familia lo conversado es muy eficaz, pero siento que en algún momento mis compañeros o profesores no me entienden, y tengo que repetir o tratar de hacerles entender lo que les quiero decir" (Entrevistado 1)

"La mayoría de las veces lo toman bien, siento que entienden lo que quiero decirles, tanto mis papas, como mis amigos" (Entrevistado 2)

Otro de los factores que se emergen en las habilidades comunicativas son la transmisión del mensaje los entrevistados la definen como una parte importante de lo que se quiere transmitir sin importar el medio por que lo hagan, los entrevistados manifiestan

"Ah ya profe, pues pienso que todos los elementos son importantes porque se enlazan el uno con el otro, es importante poder transmitir un mensaje bien, sin importar el canal utilizado, por lo general mi canal es el celular y los mensajes de wasap, pues por ahí me comunicó con mis compañeros o amigos" (Entrevistado 1)

"Pues profe todos los elementos que nombras son importantes, pero creo que el más importante es el mensaje que se quiere transmitir, si no hay un buen mensaje, no habría comunicación" (Entrevistado 3)

Dentro del código de habilidades comunicativas está la efectividad con que se transmiten los mensajes que los entrevistados de la localidad de ciudad Bolívar quieren hacer entender, pues es imprescindible hacerse entender, para no generar molestias

“Es importante porque, se pueden resolver inconvenientes que se le presenten a uno y esto hace que sea muy afectiva y respetuosa la relación" (Entrevistado 1)

"Es muy efectiva nos comentamos todo lo bueno y lo malo, cuando tengo dudas sobre algo que me da vueltas en la cabeza les pregunto, porque estoy seguro de que me van a dar la mejor respuesta o consejo" (Entrevistado 1) 
Otro factor que destacan los entrevistados en las habilidades comunicativas es la opinión, pues indican que son escuchados y tenidos en cuenta a la hora de generar dichas conversaciones en familia, señalando

"He notado que mi opinión ya es tomada más en cuenta, antes cuando era niña casi no tenía voz, pero ahora que estoy más grande puedo expresarme mejor" (Entrevistado 4)

Por otro lado, el lenguaje no verbal que es otro factor importante para los entrevistados y que refleja libertad y confianza en ellos, pues hace parte de sus habilidades comunicativas, porque a través de la gesticulación expresan lo que desean, ellos indican

"Es la forma de cómo la gente se comunica, los gestos hacen que haya una comunican más fluida y se hagan entender más las palabras." (Entrevistado 1)

"Los gestos son importantes pues dicen mucho de las personas, con los gestos un o saben cuándo se está mintiendo y cuando no." (Entrevistado 3)

"Son importantes porque también pueden dar un mensaje diferente al verbal, entonces los gestos deben ir acorde a lo que se quiere comunicar." (Entrevistado 4)

La familia de códigos "técnicas para gestionar conflictos" hace referencia a las diversas formas que los entrevistados encontraron para dar respuesta a situaciones que se presentan en la cotidianidad, como la solución de conflictos, frente a la cual expresan

"Es necesaria la comunicación porque, se habla de lo que sucede y si es un problema se soluciona en el momento" (Entrevistado 2)

"Porque por medio de la comunicación se puede resolver inconvenientes que se presenten, como peleas, discusiones, bullying entre otras" (Entrevistado 3)

A partir de lo anterior se encuentra otro factor impórtate, las buenas relaciones que permiten entablar una relación de confianza y amor entre padres e hijos, los entrevistados de la localidad de ciudad Bolívar hacen énfasis en 
"Buena, nos reímos mucho y compartimos buenos momentos" (Entrevistado 6)

"La relación con mis padres es buena, porque hay familias peores, al menos casi no me pegan como lo hacen con algunas de mis amigas. Y con mis amigas pues, antes de la pandemia tenía muchas, pero ahora solo hablo con dos de vez en cuando, son muy divertidas y chistosas" (Entrevistado 7)

"Porque en esta edad necesitamos, buena comunicación con los padres, para que no la busquemos en los amigos y de pronto nos den malos consejos" (Entrevistado 8)

Otro factor influyente es la asertividad, los entrevistados opinan que la comunicación debe ser asertiva, pues el intercambio de ideas, sentimientos, gestos, respeto entre muchos aspectos más debe ser eficaz en las buenas relaciones, señalando

"La comunicación asertiva se debe tener todo el tiempo y no esperar a que se den los problemas graves, puede ser ya difícil de mejorar" (Entrevistado 8).

"Mi familia tiene una comunicación muy buena todos recibimos de la mejor manera los mensajes y se trata siempre de aclarar las cosas en su momento" (Entrevistado 3).

Para finalizar las emociones es factor importante en las técnicas de gestión de conflictos, en este espacio los entrevistados infieren que el estado de ánimo es indispensable para comunicar lo que sienten y no generar barreras en las relaciones, los entrevistados señalan

"Pues cuando no se maneja el enojo o la rabia se vuelve una barrera" (Entrevistado 6)

"Mmm... cuando vienen de mal genio del trabajo, no se les puede hablar, pero cuando el fin de semana vamos de paseo, pues estamos felices y nos reímos de todo. En mi la verdad, estoy como más malgeniada que antes" (Entrevistado 7)

En los anteriores relatos se logra evidenciar el enojo en los momentos de estrés o mucho trabajo, en otras circunstancias, quieren estar solos y que nadie los incomode, tal como 
sucede con el siguiente entrevistado

"Me gusta estar solo en mi cuarto, que respeten mi privacidad, a veces me enojo por tonterías. Jajajajaja" (Entrevistado 9)

\section{Codificación Axial}

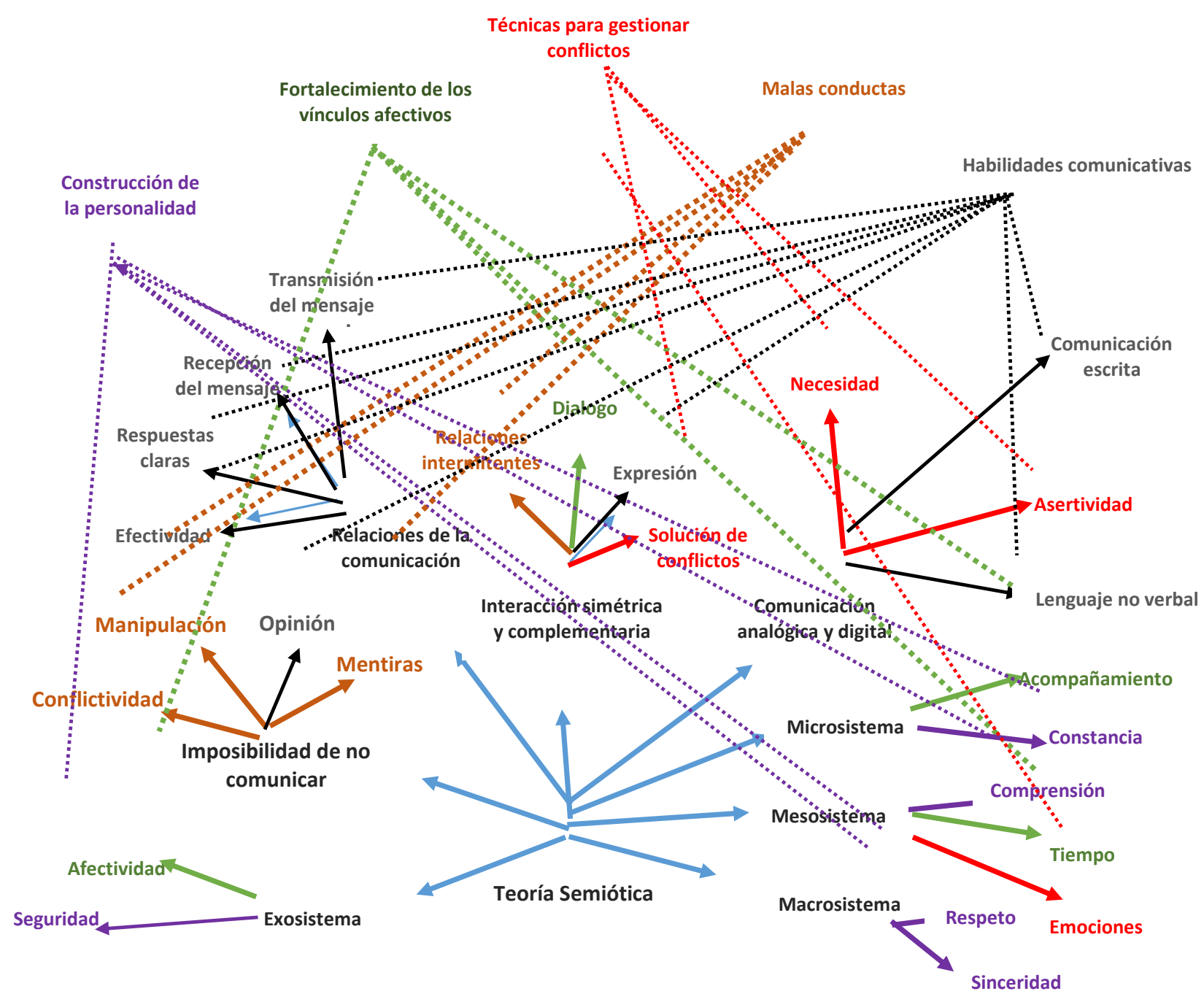

Ilustración 6. Esquema teórico de relación teoría-código

En términos generales, la ilustración 6 muestra la comunicación asertiva, expresada en la imposibilidad de no comunicar, la interacción simétrica y complementaria, la comunicación analógica y digital, axiomas de la comunicación, relacionados con el microsistema, mesosistema, exosistema, macrosistema y como estas experiencias se relacionan con los códigos, lo que permite indicar un acercamiento a la comunicación asertiva entre padres y adolescentes. 


\section{La imposibilidad de no comunicar}

Tiene bastante relación con la familia de códigos Malas conductas, pues en situaciones como la manipulación, las mentiras, las relaciones intermitentes, favorecen la conflictividad, con las cuales se obstaculiza la sana comunicación entre padre e hijos adolescentes, esto se refleja en la respuesta a la pregunta ¿Por qué los conflictos entre padres e hijos pueden dañar la comunicación?

"Porque hace que uno como hijo no quiera hablar" (entrevistado 6).

“Los conflictos cuando se llega a malas palabras, o maltrato físico, pues daña la comunicación, porque en ese momento las personas están llenas de ira y solo piensan en sí mismos" (entrevistado 8).

"Porque hace que uno como hijo no quiera hablar" (entrevistado 6)

De acuerdo con lo anterior, los resultados de esta investigación, está en desacuerdo con el aspecto de la terapia sistémica, pues permitió el abandono del clásico análisis de los procesos psíquicos intraindividuales, complementándolo con el análisis de los procesos de interacción-relación comunicacional.

\section{Relaciones de la comunicación}

Cada uno de los mensajes deben ser transmitidos y recepcionados por lo oyentes percibiendo e interpretando el mensaje, para Para Watzlawick (1985) indica que, "Lo que significa que la comunicación no es solo verbal sino también gestual - no verbal” (p.51), en la pregunta ¿Por qué los gestos son importantes a la hora de dar una opinión o mensaje asertivo?, algunas de las respuestas relevantes son

"Son importantes los gestos, ya que refuerza la comunicación, con ellos podemos saber lo que siente o piensa el que está recibiendo el mensaje" (Entrevistado No.

5)

"Los gestos son importantes pues dicen mucho de las personas, con los gestos un o saben cuándo se está mintiendo y cuando no" (Entrevistado No. 3)

Esta interrelación e interdependencia, permite aprender en el núcleo familiar técnicas para gestionar conflictos y habilidades comunicativas que se dan solo en la interacción cotidiana en el seno de la socialización primaria. Dentro de esta familia se establece importancia en 
códigos como habilidad para la transmisión y recepción del mensaje, teniendo en cuenta el lenguaje corporal y no verbal, respetar y aceptar la opinión de otros, en términos de asertividad, para lograr buenas relaciones de comunicación familiar.

\section{Interacción Simétrica y complementaria}

Ello tiene que ver directamente con los códigos de la familia construcción de la personalidad, cuando uno de los entrevistados responde a la pregunta ¿Cómo son las relaciones con sus padres y personas de su misma edad?:

"Pues con mis padres es más seria y respetuosa que con mis amigos, con ellos recochamos más" (Entrevistado 6)

Se evidencia que las relaciones de comunicación con los padres tienen una relación directa, en el proceso de desarrollo de la personalidad en la adolescencia, para llegar a formarla en la edad adulta, en ambientes protectores o familias funcionales estables y constantes en la atención de las diferentes necesidades de los menores, beneficiándose comprensión, sinceridad, confianza, respeto y seguridad, códigos relacionados con este constructo. Como microsistema la familia tiene un papel central en el desarrollo emocional y la escuela en la socialización. Esta relación es bidireccional, es decir, la forma en la que se comporten influirá en el entorno que los rodea.

\section{Comunicación analógica y digital}

La comunicación digital y analógica se complementan, ya que el aspecto digital del mensaje se codifica en función de lo analógico, lo digital hace referencia a la comunicación verbal, medible, y lo analógico a todo lo que incluye la comunicación no verbal, es decir hay que valorar tanto las palabras como otros aspectos tales como los gestos, el tono, la distancia y la posición.

De acuerdo con el anterior concepto, esta interrelación e interdependencia, permite aprender en el núcleo familiar técnicas para gestionar conflictos y habilidades comunicativas que se dan solo en la interacción cotidiana en el seno de la socialización primaria. Dentro de esta familia se establece importancia en códigos como habilidad para la transmisión y recepción del mensaje, teniendo en cuenta el lenguaje corporal y no verbal, respetar y 
aceptar la opinión y otros, en términos de asertividad, para lograr buenas relaciones de comunicación familiar. Esta clasificación de expresiones se evidencia en la entrevista a profundidad con la pregunta ¿Cómo utilizan ese tipo de comunicación? y ¿Por qué es necesaria la comunicación en la familia?

"Hablamos sobre casi todo, ellos me cuentan sus cosas y yo las mías."

"Porque en la familia, todos necesitamos de todos, y para que haya una buena convivencia." (entrevistado No. 9)

\section{DISCUSIÓN}

Como ya sabemos la comunicación es la base para tener una buena relación entre padres e hijos. pero ¿cómo lograr que entre padres e hijos se escuchen? Aunque suene un poco difícil no lo es, establecer una comunicación asertiva en las familias es posible. El diálogo asertivo permite poder expresar la opinión de forma consciente, clara, directa, con la finalidad de comunicar pensamientos e ideas desde una zona de confianza y respeto, fortaleciendo las relaciones entre los miembros de la familia.

¿En qué medida, la experiencia de uno puede ser más valiosa que en otro? ¿Cuándo el que la vive es el que es directamente afectado o beneficiado? Otra cuestión importante que surge de esta investigación es si realmente las heridas en la comunicación en la familia se pueden sanar completamente, o si dejan marcas para toda la vida y las próximas generaciones. También si hay una herencia genética que predispone a las personas a tener comportamientos agresivos en su comunicación, y si es una personalidad dominante hasta qué punto puede lograr una comunicación asertiva.

En base a lo anterior se puede inferir que hay tres formas principales en la comunicación, la forma pasiva, donde generalmente uno de los miembros no expone sus puntos de vista o preocupaciones, habla en tono de voz baja, acepta sin entender lo que se pide, llevando a que este tipo de relación sea desigual y en desventaja ante el otro miembro de la familia, causando baja autoestima y no promoviendo vínculos sanos. 
La forma de comunicación agresiva está compuesta por mensajes confusos, los cuales están conformados por gritos, regaños e incluso insultos, reflejando una comunicación distante emocionalmente entre padres e hijos, generando rechazo, miedo y resentimiento. Es así como la violencia emocional y las relaciones están constantemente en agresividad y desconfianza.

Por último, está presente la comunicación asertiva siendo la forma más recomendable pues ella implica un entorno de respeto, dialogo, y negociación. Permite fortalecer las relaciones entre padres e hijos, fomentando la autoestima en cada uno de ellos y brindando un bienestar emocional, de apoyo ante la sociedad y los posibles obstáculos que se lleguen a presentar en el adolescente, lo que permite que se sientan valorados, aceptados, en un contexto de confianza, sin cohibición, al poder entablar una conversación eficaz y saludable. Pero ¿cómo entablar estas relacione asertivas sin que intervengan la forma pasiva o agresiva?

Desarrollar una comunicación asertiva entre padres e hijos repercutirá de manera positiva en la salud emocional y mental, permitiendo ver la realidad con más claridad y entablando relaciones solidadas entre padres e hijos, enfocándose en entender el problema, opinión o sugerencia y no escuchar para luego solo contestar, ¿Cuáles son esos puntos claves dentro de la comunicación asertiva entre padres y adolescentes?

Es aquí donde ciertos aspectos deben propiciar una comunicación asertiva en familia, alguna de ellas son: evitar comparaciones, dentro de la investigación realizada algunos de los entrevistados exponían su inconformismo por que en ocasiones eran comparados con los adultos, señalando sus errores. Sin embargo, esto hacerles más que un bien, puede llegar a generar inseguridad y un sentimiento de inferioridad, haciendo que todo el tiempo ellos cojan la costumbre de compararse con los demás en diversos aspectos, si no se crea autoconfianza difícilmente se lograra una comunicación asertiva en otros ámbitos en los que se desempeñen. 
Otro aspecto relevante es ser empático la relación entre padres y adolescentes, dentro de la investigación se establecen ciertos valores que permiten una relación optima entre ellos está el respeto hacia el otro, es importante primero pensar y aclarar ideas para entablar una conversación con la otra persona, haciendo que el mensaje transmitido sea respetuoso y contundente, intentando comprender lo que piensa y siente el otro, a través de estos actos se construye la empatía en los hijos, fomentada por medio del respeto y la confianza.

Por último, la opinión es importante, una comunicación autoritaria puede limitar la comunicación asertiva, en las entrevistas de la investigación uno de ellos afirmaba que los padres mandaban y los hijos obedecían, dejando a un lado sus opiniones, esta es una forma de decirles que su voz no cuenta, es importante dejar que los hijos opinen, es la forma como ellos adoptan confianza e importancia de ser escuchados, aportando argumentos y posibles soluciones familiares.

A raíz de lo anterior sobresalta una duda que indica ¿Cómo lograr que los adolescentes expresen sus emociones?, no se puede esperar que ellos quieran que expresen sus sentimientos y pensamientos si los padres no lo hacen, es importante hablar del día a día, de sus inquietudes e intereses, escuchándolos de forma activa, de estas actitudes sobresalen los buenos consejos, alimentaran la confianza y se compartirá inquietudes, sin traspasar los límites, finalmente es el padre de familia quien debe utilizar mecanismos de comunicación asertiva para que los hijos aprendan y los implementen en su vida diaria.

Para finalizar es importante poder entender cada una de las experiencias expuestas en esta investigación, de allí parten cientos de sugerencias e inquietudes que se pueden generar a lo largo del trayecto, haciendo que as relaciones interpersonales de los jóvenes se mejoren, como también el desarrollo de su inteligencia física y emocional, son factores relevantes en la vida familiar, desarrollando en cada uno de ellos la capacidad de decir no, de pedir ayuda, favores y peticiones, expresar emociones agradables y desagradables e iniciativa para abrir, mantener y cerrar conversaciones, lo anterior permite que las comunicaciones sean favorables y con lazos familiares fuertes dentro de los hogares de la localidad de ciudad 
Bolívar. A raíz de lo anterior surge otro factor inquietante que da paso para una próxima investigación, ¿Cómo gestionan los padres el NO en los adolescentes, aceptando que tienen puntos de vistas diferentes?

\section{CONCLUSIONES}

Es fundamental en el proceso de aprendizaje desde la Infancia y la adolescencia, que tanto padres como alumnos, reciban una formación no solo en habilidades y competencias comunicativas, sino haciendo especial énfasis en el manejo adecuado del lenguaje corporal y no verbal. Esto permitiría tener más conciencia de la expresión de emociones y de la gestión adecuada de las mismas.

Las brechas generacionales no son un obstáculo, sino una oportunidad y un deber para que las nuevas generaciones formen lazos de afectivos con los adultos, pues deberían comprender que en la relación padres e hijos, debe existir unos roles y unas reglas bien claras de comportamiento, y agradecer los consejos y experiencias que se transmiten a través del diálogo y la comunicación asertiva familiar.

La etapa de la adolescencia requiere que los padres y adolescentes realicen un estudio minucioso, sobre los cambios especialmente los emocionales y en las relaciones familiares y sociales, para enfrentar el reto de orientarlos y permitir que la experiencia del adulto, combinada con la autonomía y la libertad gradual, que deben dar los padres a los hijos, los preparen realmente para ser adultos con una autoestima sana, que les permita tomar las mejores decisiones en su vida.

Para finalizar es importante tomar conciencia acerca de la importancia de la comunicación asertiva entre padres y adolescentes, pues de allí parte una serie de construcción personal enmarcadas en los valores y las buenas conductas que se generan en casa lo cual es el primer entorno en donde los adolescentes se desarrollan integralmente. 


\section{REFERENCIAS}

Aguilar-Morales, J.E. y Vargas-Mendoza, J. E. (2010) Comunicación Asertiva. Network de Psicología

Águila-Calero, G, Díaz Quiñones, J.A y Diaz- Marinez, P.M (2018). Adolescencia temprana y parentalidad. Fundamentos teóricos y metodológicos acerca de esta etapa y su manejo. Revista Medisur 15 (5) 694-700. https://www.medigraphic.com/pdfs/medisur/msu-2017/msu175o.pdf

Arango Arango, M. Z., Rodríguez, A. M., Benavides, M. S. y Ubaque, S. L. (2016). Los axiomas de la comunicación humana en Paul Watzlawick, Janet Beavin, Don Jackson y su relación con la Terapia Familiar Sistémica. Revista Fundación Universitaria Luis Amigó, 3(1), 33-50.

Bautista. N. (2011). Proceso de la Investigación Cualitativa. Bogota Colombia: El Manual Moderno.

Cáceres, B. R. (1991). Comunicación familiar. En Avances en enfermería VOL. IX No. 2 (págs. 37-48). Recuperado el 28 de 10 de 2020, de http://www.bdigital.unal.edu.co/20606/1/16768-52533-1-PB.pdf

Caridad, M., Cardeño, N., Cardeño, E., \& Castellano, M. (15 de 07 de 2017). Contribuciones de la comunicación asertiva en la resolución de conflictos dentro de instituciones de educación superior. Espacios, Vol. 38 (No 50). Recuperado el 28 de 10 de 2020, de http://www.revistaespacios.com/a17v38n50/a17v38n50p06.pdf

Crespo Comesaña, J. M (2011). Bases para construir una comunicación positiva en la familia. Revista de Investigación en Educación, 9 (2), 91-98. Http//Dialnet BasesParaConstruirUnaComunicacionPositivaEnLaFamil-4731297.pdf

Corrales, A.; Quijano, N. K. y Góngora, E. A. (2017). Empatía, comunicación asertiva y seguimiento de normas. Un programa para desarrollar habilidades para la vida . Enseñanza e Investigación en Psicología, 22(1), 58-65. https://www.redalyc.org/pdf/292/29251161005.pdf

Dirino, L. (2016). Adolescencia tiempo de crisis y transiciones. Revista Ciencias de la Educación 26 (47), 258-270 http://servicio.bc.uc.edu.ve/educacion/revista/47/art16.pdf

Dorys, Ortiz G. (2008) La terapia familiar sistémica. Quito. Ecuador. 48 - 49. Recuperado de:

https://dspace.ups.edu.ec/bitstream/123456789/6096/1/Terapia\%20familiar\%20sist emica.pdf 
Gaete, V.. (2015). Desarrollo psicoscial del adolescente. Revista Chilena de Pediatria, 86 (3). https://scielo.conicyt.cl/scielo.php?script=sci_arttext\&pid=S037041062015000600010

Gumes-Hidalgo, M. Gonzalez-Fierro, M.J y Vicario, H. (2017). Desarrollo durante la adolescencia, Aspectos fisicos, psicologicos y sociales. RevistaPediatria. https://www.adolescenciasema.org/ficheros/PEDIATRIA\%20INTEGRAL/Desarrollo\% 20durante\%20la\%20Adolescencia.pdf

Gaeta González,L. y Galvanovskis Kasparane, A (2009). Asertividad: Un análisis teórico empírico Revista Enseñanza e Investigación en Psicología Volumen 14 (2) 403-425. https://www.redalyc.org/pdf/292/29211992013.pdf

Gonzalez-Vidales, C. (2015). Comunicación y sociedad. Revista Historia, teoría e investigación de la comunicación Volumen 8 (1)pp. 1 - 27.

http://www.scielo.org.mx/scielo.php?script=sci_arttext\&pid=S0188252X2015000100002

Guardia de Viggiano, N.V (2019). Lenguaje y comunicación. Coordinación educativa y cultural centroamerica 25, 6-140.

https://ceccsica.info/sites/default/files/content/Volumen_25.pdf

Gonzalo Musitu, Sofía Buelga. (2006). La comunicación familiar desde la perspectiva sistémica (Capítulo publicado en el libro "Psicología de la Comunicación". Madrid Pirámide. 4 - 19. Recuperado de https://www.avntf-evntf.com/wpcontent/uploads/2016/06/Lacomunicacionfamdesdeperspectsist.GMusitu.pdf

Hernandez. S (2006). Metodologia de la Investigación. Mexico: McGraw Hill.

Ministerio de Salud. (04 de octubre de 1993). Resolución Número 8430 de 1993. Santafé de Bogotá D.C., Colombia. Recuperado el 15 de 12 de 2020, de https://www.minsalud.gov.co/sites/rid/Lists/BibliotecaDigital/RIDE/DE/DIJ/RESOLUC ION-8430-DE-1993.PDF

Organizacional. México: Asociación Oaxaqueña de Psicología A.C.

http://eoepsabi.educa.aragon.es/descargas/G_Recursos_orientacion/g_5_habilidad es_sociales/g_5.3.hhss_asertivas/1.6.Comunicacion\%20asertiva.pdf 